\title{
DNA Replication-Initiation Proteins in Eukaryotic Cells
}

\author{
Aftab Amin ${ }^{1,2}$, Cheung Man Hei ${ }^{1,2}$ and Chun Liang ${ }^{1-4 *}$ \\ and Technology, Hong Kong, China \\ ${ }^{2}$ HKUST Fok Ying Tung Research Institute, Guangzhou, China \\ ${ }^{3}$ Intelgen Limited, Hong Kong-Guangzhou-Foshan, China \\ ${ }^{4}$ EnKang-EnZhi, Limited, China
}

${ }^{1}$ Division of Life Science, Center for Cancer Research and State Key Lab for Molecular Neuroscience, Hong Kong University of Science

*Corresponding author: Chun Liang, Division of Life Science, Center for Cancer Research and State Key Lab for Molecular Neuroscience, Hong Kong University of Science and Technology, Hong Kong, China

\section{ARTICLE INFO}

Received: 幽 November 01, 2019

Published: 仹 November 18, 2019

Citation: Aftab Amin, Cheung Man Hei, Chun Liang. DNA Replication-Initiation Proteins in Eukaryotic Cells. Biomed J Sci \& Tech Res 23(1)-2019. BJSTR. MS.ID.003829.

Keywords: DNA replication; DNA replication-initiation protein (DRIP); Replication licensing; Pre-replicative complex (pre-RC); Ribosome biogenesis; Cell cycle; Origin recognition complex (ORC); Noc3p; Ipi3p; Cdc6p; Cdt1p; MCM proteins

\begin{abstract}
DNA replication is a highly regulated cellular process in proliferating cells, involving cell cycle dependent assembly of DNA replication-initiation proteins (DRIPs) onto origins of replication. The process of pre-replicative complex (pre-RC) formation at the $\mathrm{M}$-to- $\mathrm{G}_{1}$ transition, also known as replication licensing, requires origin recognition complex (Orc1-6p) that binds and marks replication origins to facilitate the loading of additional DRIPs, such as Noc3p, Ipi3p, Cdc6p, Cdt1p and Mcm2-7p. The subsequent activation of pre-RC at the $\mathrm{G}_{1}$-to-S transition is dependent upon cyclin-dependent kinases (CDKs) and Dbf4-dependent kinase (DDK). This sequential process ensures that DRIPs are precisely loaded to form pre-ICs and then activated by their regulators so that chromosomal DNA is replicated only once per cell cycle. Despite substantial gains in the study of the mechanisms and regulation of pre-RC, the finite details of the pre-RC assembly and disassembly processes remain unclear and controversial. In this review we describe the present state of understanding on DRIPs and the pre-RC architecture and dynamics.
\end{abstract}

Abbreviations: CDC6: Cell Division Cycle; CDC14: Cell Division Cycle; CDC45: Cell Division Cycle; CDT1: Cdc10-Dependent Transcript; CMG: Cdc45p-MCM-GINS; GINS: Slf5, Psf1, Psf2, Psf3; IPI3: Involved in processing IST2; MCM: Mini-chromosome maintenance; NOC3: Nucleolar Complex; ORC: Origin Recognition Complex; Pol30/ PCNA: Proliferating Cell Nuclear Antigen; pre-IC: Pre-Initiation Complex; pre-RC: PreReplicative Complex; DRIP: DNA Replication-Initiation Proteins

\section{Introduction}

The DNA double helix structure was first proposed by J.D. Watson \& F. Crick [1]. They also presented a semi-conservative hypothesis, which necessitated that two single strands of a DNA double helix separate and each acts as a template for the synthesis of a new strand of DNA [2]. This semi-conser vative model of DNA replication was later confirmed by experiments (Meselson \& Stahl 1958). The replicon model for the control of DNA replication first proposed by Jacob, Cuzin and Brenner states that a trans-acting regulatory factor, the initiator protein specifically binds onto the cisacting sequence, known as the replicator at a specific chromosomal location to sequester other replication factors to initiate replication of the replicon at the origin of replication [3]. The replicon model therefore formed the basis of DNA replication studies that have been carried out in model organisms such as bacteria, budding yeast, fission yeast and metazoans.

Recent advances in molecular and cellular biology, genetics and biochemistry have resulted in the identification of dozens of DNA replication-initiation proteins (DRIPs) and their regulators that control the initiation of DNA replication. However, these discoveries present new challenges and clearly indicate that there are still many aspects of DNA replication initiation that remain elusive. The initiation of eukaryotic DNA replication is a two-step process involving replication licensing and origin activation. Pre-replication complex (pre-RC) assembly at the $\mathrm{M}-\mathrm{to}-\mathrm{G}_{1}$ transition (replication/ 
origin licensing) is stringently controlled, contributing to the onceper-cell cycle control of DNA replication [4-12]. Deregulated replication may lead to aneuploidy, cancer and/or cell death [13-17].

The pre-replication complex is activated by cyclin-dependent kinases (CDKs) and Dbf4p-dependent kinase (DDK) after the cell has passed the restriction point (START in yeast), to form a preinitiation complex (pre-IC), at an origin of replication [18-20]. Reformation of the pre-RC within the same cell cycle is prevented by the phosphorylation (inactivation) of certain pre-RC factors $[21,22]$. Some other components are exported from the nucleus or degraded [23]. Origin activation is therefore irreversible once the cell becomes committed to the cell cycle after passing the restriction point. Activation of the origin leads to the action of helicases unwinding the DNA double helix and forming replication forks. Replication proteins assembled at replication forks incorporate nucleotides into newly synthesized DNA chains. This is done with high fidelity to ensure that replication results in viable genomic duplication.

\section{Eukaryotic Replication-Initiation Proteins (DRIPs)}

Eukaryotic DNA replication has been most extensively studied in budding yeast Saccharomyces cerevisiae [4,5,9,24]. Most of the eukaryotic DRIPs were first discovered in budding yeast, and their homologs in metazoans were then identified by sequence homology. Budding yeast cells replicate their DNA from defined origins of replication that were first defined as autonomously replicating sequences (ARSs). The hetero-hexameric origin recognition complex (ORC) and Noc3p (nucleolar complex-associated protein) are bound to chromatin throughout the cell cycle, forming the postreplicative complex (post-RC) during $S, G_{2}$ and most of $M$ phases of the cell cycle [9,12,22,24-27].

After dephosphorylation of DRIPs such as ORC, Cdc6p and Mcm3p by Cdc14p during the $M-$ to $-\mathrm{G}_{1}$ transition, the sequential loading of Ipi1-3p, Cdc6p, Cdt1p, and MCM proteins at replication origins establishes the pre-replication complex (pre-RC) $[9,22,25,27,28]$. This process of origin licensing is followed by origin activation [18-20].

\section{Origin Recognition Complex (ORC)}

Yeast proteins were fractionated to identify DRIPs, and this led to the discovery of Orc1-6p as a hetero-hexameric protein complex, which was characterized and identified as an ATP-dependent ARS binding protein complex [29,30]. Components of ORC are conserved in eukaryotes, and this facilitated the identification of ORC in other eukaryotic organisms. It is evident now that ORC binds and protects replication origins throughout the cell cycle and is essential for loading other DRIPs onto replication origins [6,10,13,23,26,2830]. However, the dynamics of ORC at replication origins during DNA replication still remains to be elucidated. All ORC subunits barring Orc6p belong to the AAA+ ATPase super family of ring shaped P-loop NTPases. These proteins bind and hydrolyze ATP to facilitate energy-dependent processes such as remodeling of macromolecules or translocation. Orc1-5p form the critical sub complex that possess DNA binding affinity, but Orc1-6p together, are essential for the assembly and maintenance of pre-RCs.

Orc6p is directly involved in the origin association of Cdt1pMcm2-7p [23,32-35]. Some reports have suggested that multiple MCM subunits have direct interactions with ORC during the loading of Cdt1p-Mcm2-7p [9,24,36]. Recent cryo-electron microscopy (cryo-EM) and single molecule studies using purified proteins show that the architecture of the Orc1-6p complex is asymmetric, while the Mcm2-7p complex forms a symmetric double-hexamer during pre-RC formation [36-43]. These interesting structural characteristics have spurred debates in the DNA replication field with several different models being proposed [8,38,40-44].

The "One-ORC" model proposes that a single ORC hexamer recruits one $\mathrm{Mcm} 2-7 \mathrm{p}$ single hexamer which in turn recruits the second Mcm2-7p complex [10,41,44]. The "two-ORC" model suggests that two Mcm2-7p complexes are loaded onto origins in the same manner by two ORC single-hexamers, each bound at a single origin [43]. This is based on the finding that increasing ORC concentrations lead to a sigmoidal, rather than linear, increase in MCM loading and that the presence of two ORC binding sites supports MCM loading 10-fold more efficiently than a single ORC binding site [43]. It is interesting to note that most cryo-EM studies support the one ORC model, while there is evidence for the existence of ORC dimers, stated as $<10 \%$ of the purified ORC [37]. Our recent protein interaction study of budding yeast and human DRIPs shows that several yeast and human ORC subunits self-interact [24].

Our study also suggests that ORC single-hexamers dimerize to form double-hexamers at replication origins before MCM loading at the M-to-G1 transition in vivo (unpublished). Importantly, our study suggests that the ORC dimer separates into two single-hexamers, each binding and protecting one of the two newly replicated origins from histone invasion. These findings uncovered an essential, cell cycle-dependent, and likely semi-conservative 'dimerization cycle' of ORC that regulates DNA replication.

\section{Noc3p}

Noc3p is a basic helix-loop-helix (bHLH) protein that was initially identified as a component of the heterodimeric Noc2pNoc3p complex involved in ribosome biogenesis in budding yeast [45-47]. This complex is involved in the formation of the $40 \mathrm{~S}$ ribosomal subunits and its nuclear export [45]. Studies have indicated that the Noc complex mediates the maturation of $60 \mathrm{~S}$ ribosomal precursors and the intra-nuclear transport of the $60 \mathrm{~S}$ ribosome subunit [45]. Noc3p's essential role in DNA replication has been established in budding yeast, where it is required for the chromatin association of Cdc6p and MCM proteins during pre-RC formation [25]. Noc3p is conserved in eukaryotic organisms, and the human homolog FAD24/hNOC3 is involved in DNA replication, differentiation and adipogenesis [48-52,12]. 
Noc3p in both budding yeast and human cells interacts with ORC, Cdt1p, MCM and other DRIPs, and is required for the recruitment of Ipi3p (involved in processing IST2), Cdc6p and MCM proteins to replication origins for pre-RC formation during the M-to- $G_{1}$ transition $[9,12,24,25]$. Both human and budding yeast Noc3p binds chromatin at replication origins throughout the cell cycle $[12,25]$. Silencing or depletion of human or budding yeast Noc3p resulted in pre-RC formation failure, S-phase entry defects and eventual cell death, without affecting the cellular levels of other known DRIPs within the experimental timeframe [12,25]. Overexpression of Noc3p results in dosage lethality in Cdc6p mutant yeast cells, just as overexpression of other DRIPs do, which further elaborates Noc3p's role as a DRIP [54]. The specific inhibition of RPA194 (a ribosome biogenesis pathway protein) did not result in pre-RC assembly defects, indicating a separation of function similar to that reported in the budding yeast $[12,25]$.

Results from fission yeast experiments demonstrating significantly impaired S-phase entry and cell cycle progression with eventual $\mathrm{G}_{2} / \mathrm{M}$ phase arrest (likely due to incompletion DNA replication) following Noc3p depletion (inevitably incompletion depletion), similar to the phenotypes observed following ORC or MCM depletion, are consistent with current and previous findings regarding Noc3p's role in DNA replication [12,25,54]. Noc3p is therefore a conserved, multifunctional protein. In addition to Noc3p, ORC, Ipi3p and Cdc6p have also been implicated in ribosome biogenesis, as well as DNA replication, suggesting that the processes of DNA replication and ribosome biogenesis are intricately linked and coordinated [10,13,26,28,46,56].

\section{Ipi3p}

Ipi3p is a component of the Rix1 complex (Ipi1p-Ipi2p/Rix1pIpi3p), which is required for processing of ITS2 sequences from 35S pre-rRNA and ribosome biogenesis [56-58]. A yeast functional proteomic screen identified Ipi1p, $-2 p$ and $-3 p$ as novel DRIPs [9]. Ipi3p binds chromatin at ARS sequences in an ORC-, Noc3p-and cell cycle-dependent manner [9]. Ipi3p is required for loading Cdc6p Cdt1p and MCM2-7p onto chromatin during the M-to- $\mathrm{G}_{1}$ transition and also for the maintenance of the pre- $R C$ in $G_{1}$ phase [9]. Ipi3p is therefore required for pre-RC assembly and maintenance, and this is independent of its role in ribosome biogenesis. Interestingly, as Ipi3 also interacts with Noc3p, it serves to connect ORC and Noc3p to the other pre-RC components [9,24]. Like Noc3p and other DRIPs, Ipi3p is also conserved in eukaryotes, including humans $[24,27]$. The Ipi3p homolog in fission yeast crb3 was reported to interact with cut5 (a protein involved in DNA replication and check point control) [59].

Interestingly the cut5 homolog in budding yeast (Dpb11p) is required for replication initiation after pre-RC assembly $[60,61]$. Our study has established human hIPI3 as being required for human DNA replication licensing, cell cycle progression and cell proliferation [27]. Like the budding yeast Ipi3p, hIPI3 interacts with several human ORC and MCM subunits [27]. In particular, the interactions of hIPI3 with hORC2, hNOC3 and hMCM2 are consistent with those previously observed in budding yeast $[9,27]$. Furthermore, hIPI3, like hNOC3p, also preferentially associates with known human replication origins [27]. hIPI3 silencing experiments also suggest that hIPI3 role in DNA replication is independent of its role in ribosome biogenesis.

\section{Cdc6p and Cdt1p}

Cdc6p has been extensively studied in budding yeast. The protein belongs to the AAA+ ATPase superfamily and shares a high degree of homology with Orc1p [62]. Deregulation of this protein in humans has been linked to tumorigenesis [63,64]. Cdc6p functions as a DRIP by facilitating Mcm2-7p chromatin binding [28]. Mutations in the Cdc6p ATP binding domain significantly abrogate chromatin binding [65]. Defective Cdc6p ATP hydrolysis results in Mcm2-7p loading failure [65]. Furthermore, Cdc6p is also involved in the maintenance and activation of the checkpoint mechanisms in the cell cycle by coordinating the $\mathrm{S}$ and $\mathrm{M}$ phases of the cell cycle [66]. When over-expressed, Cdc6p in S. pombe can induce several rounds of DNA replication without cell division [67]. Furthermore, CDC6 gene expression is cell cycle dependent. Cdc6p accumulates during late $M$ and $G_{1}$ phases of the cell cycle, owing to the low Cdc28p-Clb5p-Clb6p CDK activity [68].

Cdc6p is degraded at the onset of S phase mediated by CDK phosphorylation, triggering proteolysis through the SCF/Cdc4 pathway $[69,70]$. This mechanism contributes to the once-per-cell cycle control of DNA replication [68]. Structure studies have shown that Cdc6p binding to the DNA-bound ORC forms the ORC-Cdc6pDNA complex [38,71]. EM studies suggest that Cdc6p closes the crescent shaped ORC structure, forming a ring [72]. This process renders a conformational change in ORC which facilitates Mcm2$7 p$ loading [10]. Cdt1p (Cdc10-dependent transcript) was first identified in S. pombe as having Cdc10p dependent, cell cycle regulated expression [73]. It was later characterized as a key licensing factor in pre-RC formation in Xenopus [74].

Cdt1p is required for MCM nuclear localization and subsequent chromatin loading in budding yeast [75,23]. Cdt1p depletion abrogates origin firing, but does not affect $S$ phase progression [76]. CDT1 expression is at its highest in $\mathrm{G}_{1}$ phase followed by a steady decline upon entry into $S$ phase in human cells during [77]. In budding yeast, Cdt1p accumulates in $G_{1}$ phase and is subsequently exported into the cytoplasm before the START checkpoint [76]. A number of cryo-EM studies using purified proteins have revealed the complex structure of Cdt1p together with ORC, Cdc6p and MCM proteins $[36,42,54,78,79]$. Further in vitro and in vivo studies are required to fully elucidate the structure and interactions of these complexes.

\section{Minichromosome Maintenance (MCM) Proteins}

Originally identified through a genetic screen for factors involved in plasmid maintenance in budding yeast [80], the $\mathrm{Mcm} 2-$ $7 \mathrm{p}$ complex comprise of six conserved, structurally related proteins 
[80-82]. The MCM complex is a critical component of the pre-RC and is involved in replication initiation and elongation [83]. $\mathrm{Mcm} 2-$ $7 p$ chromatin association occurs during late M to early G1 phase, where it migrates with the replication fork in S phase in accordance with its function as a putative DNA helicase [84]. It has been shown that a complex of Cdc45p-Mcm2-7p-GINS (CMG) is the active helicase $[85,86]$.

Regulation of the MCM complex is controlled by the nuclear export sequence (NES) within Mcm3p and the nuclear localization sequence (NLS) found in Mcm2p and Mcm3p [87]. These sequences are in turn regulated by B-type cyclin-CDK phosphorylation and Cdc14p dephosphorylation $[22,87,88]$. Mcm2-7p belong to the AAA+ ATPase family of proteins. However, none of the subunits actually exhibit any ATPase activity as determined by in vitro studies [89]. MCM complex subunits interact with one another to form a head-tail hetero-hexamer $[9,24,89,82]$.

Cryo-EM studies have now shed further light onto the structure of the MCM complex. A study identified that interlocked aminoterminal interactions form a central channel within the MCM double-hexamer [40]. This channel, comprising of four concentric rings consisting of interior $\beta$-hairpins, creates a passage specifically for duplex DNA [40]. The passage is flanked by two pairs of gateforming $\mathrm{Mcm} 2 \mathrm{p}$ and Mcm5p subunits [40]. Although each MCM subunit has unique features and functions, the DNA channel structure forms the catalytic core of the MCM dimer in the budding yeast replicative helicase $[11,42]$.

\section{Pre-Initiation Complex and Proteins at Replication Forks}

After pre-RC formation, CDK and DDK promote the loading of Mcm10p, Cdc45p, Sld2p, Sld3p, Dpb11p, GINS and DNA polymerases to the pre- $\mathrm{RC}$, hence forming the pre-initiation complex (pre-IC) These actions are followed by helicase activation and the unwinding of replication origins [84,90-92].

Mcm10p was initially identified through genetic screening [80]. Mcm10p has since been shown to be a requisite for DNA replication initiation and elongation through its interactions with the pre-RC components in budding yeast [93,94]. Mcm10p chromatin association is cell cycle regulated [95]. The Mcm2-7p complex recruits $\mathrm{Mcm} 10 \mathrm{p}$ to origins where it coordinates DDK phosphorylation of MCM, resulting in helicase activation [96]. Mcm10p deficiency in budding yeast primarily causes defects in replication fork progression [94]. Studies further implicate that Mcm10p recruits Cdc45p and Pol $\alpha /$ primase to origins [97]. Mcm10p is also a component of the replisome progression complex (RPC) and migrates with the replication fork [98]. Furthermore, Mcm10p recruits Sld3p and Cdc45p to the pre-RC [97]. Sld3p and GINS competitively bind Mcm2-7p [99]. Sld3p dissociates from the MCM complex following DNA unwinding, facilitating GINS-MCM complex interactions for CMG complex formation [99,94].
Subsequently, replication factor A (RFA) binds to protect the single stranded DNA, and new DNA strands are synthesized by DNA polymerases facilitated by other replication proteins. Given the significant advances in our understanding of replication initiation and replication fork progression, future investigations into the mechanisms and regulation of these proteins in normal and cancer cells may lead to discoveries that have significant clinical value.

\section{Concluding Remarks}

Eukaryotic genome duplication entails an intricately coordinated process in which the cell enforces a series of steps to ensure the regulated assembly and disassembly of several protein complexes at replication origins. Although there has been significant progress in elucidating the DNA replication initiation control mechanisms, several key questions remain unanswered. At present, several models of DNA replication licensing have been proposed, which although similar in principle, have fundamental differences. For example, the ORC dynamics presents a fundamental dilemma. Is "one-ORC" sufficient to load two MCM single-hexamers in vivo? Or are "two-ORCs" required to allow for the symmetrical and efficient loading of pre-RC proteins? How are new ORC proteins recruited to bind and protect the newly replicated origins? Our recent in vivo data suggest that ORC self-interacts and dimerizes to from doublehexamers before MCM proteins are loaded onto replication origins. This process is likely mediated by other DRIP(s) (unpublished). Our model of ORC dimerization presents an essential, cell-cycle dependent and semi-conservative ORC dimerization cycle, in a dynamic cellular system to ensure faithful DNA replication.

Although it has been established that DRIPs are recruited sequentially onto replication origins, their respective biochemical activities have yet to be fully elucidated. For example, most DRIPs are known to bind ATP and have ATP hydrolysis activities. However, how ATP binding and hydrolysis are regulated to facilitate the formation and dissolution of DRIP complexes is not clear.

Another issue concerns the roles of ribosome biogenesis proteins in DNA replication initiation. Ample evidence from studies in yeast and human cells support the dual roles of proteins like Noc3p, Ipi3p, Yph1p and Cdc6p in DNA replication and ribosome biogenesis. Importantly, we have identified separation-of-function Noc3p mutants that are defective in DNA replication without obvious defects in ribosome biogenesis, and vice versa (unpublished). These findings suggest that DNA replication and ribosome biogenesis are coordinated by these multi-functional proteins.

DNA replication is stringently controlled to ensure the integrity of genetic information in cell proliferation. Deregulation of DNA replication may give rise to cancer, for example, the deregulation of Cdt1p and Cdc6p promotes re-firing of the same origin [100]. As a result of active DNA replication and cell division, pre-RC proteins such as Cdc6p and Cdt1p and MCM complex proteins are found to be overexpressed in cancers to promote cancer development, 
invasion and metastasis $[101,102]$. Importantly, it has been shown that silencing of DRIPs results in apoptosis of cancer cells but not normal cells [103-113]. It is therefore important to study DNA replication, particularly DRIPs, to further understand the molecular pathways and mechanisms involved in cancer development, helping to develop novel cancer detection methods and therapeutics.

\section{Acknowledgment}

The work from our lab was supported by the Foshan Science and Technology Bureau, Guangdong, China (2015IT100132), Department of Science and Technology of Guangdong Province, China (2017B050506003), Guangzhou Committee of Science and Information Innovation, China (201604020038), Innovation and Technology Fund, Hong Kong, China (GHX/002/17GD), and Science and Technology Innovation Bureau of Guangzhou Development District, Guangdong, China (CY2019-005).

\section{References}

1. Watson JD, Crick F (1953) Molecular structure of Nucleic Acids: A Structure for Deoxyribose Nucleic Acid Nature 171: 737-738.

2. Watson JD, Crick F (1953) Genetic implications of the structure of deoxyribonucleic acid. Nature 171: 964-967.

3. Jacob F, Brenner S, Cuzin F (1963) On the regulation of DNA synthesis in bacteria: the hypothesis of the replicon. Cold Spring Harb Symp Quant Biol 256: 298-300.

4. Bell SP, Dutta A (2002) DNA replication in eukaryotic cells. Annu Rev Biochem 71: 333-374.

5. Méndez J, Stillman B, (2003) Perpetuating the double helix: molecular machines at eukaryotic DNA replication origins. Bioessays 25: 11581167.

6. Sclafani RA, Holzen TM (2007) Cell cycle regulation of DNA replication. Annual Review of Genetics 41: 237-280.

7. Araki H (2011) Initiation of chromosomal DNA replication in eukaryotic cells; contribution of yeast genetics to the elucidation. Genes Genet Syst 86: 141-149.

8. Diffley JF (2011) Quality control in the initiation of eukaryotic DNA replication. Philos Trans R Soc Lond B Biol Sci 366: 3545-3553.

9. Huo L, Wu R, Yu Z, Zhai Y, Yang X, et al. (2012) The Rix1 (Ipi1p-2p$3 p)$ complex is a critical determinant of DNA replication licensing independent of their roles in ribosome biogenesis. Cell Cycle 11(7): 1325-1339.

10. Bell PS, Labib K (2016) Chromosome Duplication in Saccharomyces cerevisiae. Genetics 203(3): 1027-1067.

11. Zhai Y, Li N, Jiang H, Huang X, Gao N, et al. (2017) Unique roles of the non-identicle MCM subunits in DNA replication licensing. Mol Cell 67(2): 168-179.

12. Cheung MH, Amin A, Wu R, Qin Y, Zou L, et al. (2019) Human NOC3 is essential for DNA repliation licensing in human cells. Cell Cycle 18(5): 605-620.

13. Hook SS, Lin JJ, Dutta A (2007) Mechanisms to control rereplication and implications for cancer. Curr Opin Cell Biol 19(6): 663-671.

14. Shima N, Alcaraz A, Liachko I, Buske TR, Andrews CA, et al. (2007) A viable allele of Mcm4 causes chromosome instability and mammary adenocarcinomas in mice. Nature Genetics 39(1): 93-98.

15. Rakotomalala L, Studach L, Wang WH, Gregori G, Hullinger RL, et al. (2008) Hepatitis B virus X protein increases the Cdt1-to-Geminin ratio inducing DNA re-replication and polyploidy. J Biol Chem 283(42): 28729-28740.
16. Kayes JO, Loddo M, Patel N, Patel P, Minhas S, et al. (2009) DNA replication licensing factors and aneuploidy are linked to tumor cell cycle state and clinical outcome in penile carcinoma. Clin Cancer Res 15(23): 73357344.

17. Mahadevappa R, Neves H, Yuen SM, Jameel M, Bai Y, et al. (2018) DNA Replication Licensing Protein MCM10 Promotes Tumor Progression and Is a Novel Prognostic Biomarker and Potential Therapeutic Target in Breast Cancer. Cancers (Basel) 10(9): 282.

18. Sheu YJ, Stillman B (2010) The Dbf4-Cdc7 kinase promotes S phase by alleviating an inhibitory activity in Mcm4. Nature 463(7277): 113-117.

19. Heller RC, Kang S, Lam WM, Chen S, Chan CS, et al. (2011) Eukaryotic origin-dependent DNA replication in vitro reveals sequential action of DDK and S-CDK kinases. Cell 146(1): 80-91.

20. Yeeles JT, Deegan TD, Janska A, Early A, Diffley JF (2015) Regulated eukaryotic DNA replication origin firing with purified proteins. Nature 519(7544): 431-435.

21. Nguyen VQ, Co C, Li JJ (2001) Cyclin-dependent kinases prevent DNA re-replication through multiple mechanisms. Nature 411(6841): 10681073.

22. Zhai Y, Yung PYK, Huo L, Liang C (2010) Cdc14p resets the competency of replication licensing by dephosphorylating multiple initiation proteins during mitotic exit in budding yeast. J Cell Sci 123: 3933-3943.

23. Wu R, Wang J, Liang C (2012) Cdt1p, through its interaction with Mcm6p, is required for the formation, nuclear accumulation and chromatin loading of the MCM complex. J Cell Sci 125(Pt 1): 209-219.

24. Wu R, Amin A, Wang Z, Huang Y, Cheung MH, et al. (2019) The Interaction networks of the budding yeast and human DNAreplication-initiation proteins. Cell Cycle 18(6-7): 723-741.

25.Zhang Y, Yu Z, Fu X, Liang C (2002) Noc3p, a bHLH protein, plays an integral role in the initiation of DNA replication in budding yeast. Cell 109(7): 849-860

26. DePamphilis ML (2003) The 'ORC cycle': a novel pathway for regulating eukaryotic DNA replication. Gene 310: 1-15.

27. Huang S, Xu X, Wang G, Lu G, Xie W, et al. (2016) DNA replication initiator Cdc6 also regulated ribosomal DNA transcription initiation. Journal of Cell Science 129(7): 1429-1440.

28. Liang C, Stillman B (1997) Persistent initiation of DNA replication and chromatin-bound MCM proteins during the cell cycle in cdc6 mutants. Genes Dev 11(24): 3375-3386.

29. Bell PS, Stillman B (1992) ATP-dependent recognition of eukaryotic origins of DNA replication by a multiprotein complex. Nature 357(6374): 128-134.

30. Bell SP, Kobayashi R, Stillman B (1993) Yeast origin recognition complex functions in transcription silencing and DNA replication. Science 262(5141): 1844-1849.v

31. Weinreich M, Liang C, Stillman B (1999) The Cdc6p nucleotide binding motif is required for loading $\mathrm{mcm}$ proteins onto chromatin. Proc Natl Acad Sci USA 96(2): 441-446.

32. Semple JW, Da-Silva LF, Jervis EJ, Ah-Kee J, Al-Attar H, et al. (2006) An essential role for Orc6 in DNA replication through maintenance of prereplicative complexes. EMBO J 25(21): 5150-5158.v

33. Chen S, de Vries, MA, Bell SP (2007) Orc6 is required for dynamic recruitment of Cdt1 during repeated $\mathrm{Mcm} 2-7$ loading. Genes Dev 21(22): 2897-2907.

34. Da-Silva L, Duncker BP (2007) ORC Function in Late G1: Maintaining the Licensing for DNA Replication. Cell Cycle 6(2): 128-130.

35. Bleichert F, Botchan MR, Berger JM (2015) Crystal structure of the eukaryotic origin recognition complex. Nature 519(7543): 321-326.

36. Yuan Z, Riera A, Bai L, Sun J, Nandi S, et al. (2017) Structural basis of Mcm2-7 replicative helicase loading by ORC-Cdc6 and Cdt1. Nature Structural \& Molecular Biology 24(3): 316-324. 
37. Sun J, Kawakami H, Zech J, Speck C, Stillman B, et al. (2012) Cdc6-induced conformational changes in ORC bound to origin DNA revealed by cryoelectron microscopy. Structure 20(3): 534-544.v

38. Sun J, Evrin C, Samel SA, Fernández-Cid A, Riera A, et al. (2013) Cryo-EM structure of a helicase loading intermediate containing ORC-Cdc6-Cdt1MCM2-7 bound to DNA. Nat Struct Mol Biol 20(80: 944-951.v

39. Sun J, Fernandez-Cid A, Riera A, Tognetti S, Yuan Z, et al. (2014) Structural and mechanistic insights into Mcm2-7 double-hexamer assembly and function. Genes \& Dev 28: 2291-2303.

40. Li N, Zhai Y, Zhang Y, Li W, Yang M, et al. (2015) Structure of the eukaryotic MCM complex at 3.8 angstrom Nature 524: 186-191.

41. Ticau S, Freidman LJ, Ivica NA, Gelles J, Bell SP (2015) Single-molecule studies of origin licensing reveal mechanisms ensuring bidirectional helicase loading. Cell 161(3): 513-525

42. Zhai Y, Cheng E, Wu H, Li N, Yung PYK, et al. (2017) Open-ringed Structure of the Cdt1-Mcm2-7 Complex as a Precursor of the MCM Double-hexamer. Nature Struct Mol Biol 24(3): 300-308.

43. Coster G, Diffley JFX (2017) Bidirectional eukaryotic DNA replication is established by quasi-symemetrical helicase loading. Science 357(6348): 31-318.

44. Warner MD, Azmi IF, Kang S, Zhao Y, Bell SP (2017) Replication originflanking roadblocks reveal origin-licensing dynamics and altered sequence dependence. J Biol Chem 292(52): 21417-21430.

45. Milkereit P, Gadal O, Podtelejnikov A, Trumtel S, Gas N, et al. (2001) Maturation and intranuclear transport of pre-ribosomes requires Noc proteins. Cell 105(4): 499-509.

46. Dlakić M, Tollervey D (2004) The Noc proteins involved in ribosome synthesis and export contain divergent HEAT repeats. RNA 10(3): 351354.

47. Bassler J, Kallas M, Hurt E (2006) The NUG1 GTPase reveals and $\mathrm{N}$-terminal RNA-binding domain that is essential for association with 60 S pre-ribosomal particles. J Biol Chem 281(34): 24737-24744.

48. Johmura Y, Osada S, Nishizuka M, Imagawa M (2008) FAD24 acts in concert with histone acetyltransferase $\mathrm{HBO} 1$ to promote adipogenesis by controlling DNA replication. J Biol Chem 283(4): 2265-2274.

49. Johmura Y, Osada S, Nishizuka M, Imagawa M (2008) FAD24, a regulator of adipogenesis, is required for the regulation of DNA replication in cell proliferation. Biol Pharm Bull 31(6): 1092-1095.

50. Tominaga K, Johmura Y, Nishizuka M, Imagawa M (2004) Fad24, a mammalian homolog of Noc3p, is a positive regulator in adipocyte differentiation. J Cell Sci 117: 6217-6226.

51. Lei T, Bi Y, Gao MJ, Gao SM, Zhou LL, et al. (2013) HES1 inhibit adipogenesis of porcine mesenchymal stem cells via transcriptional repression of FAD24. Domest Anim Endocrinol 45(1): 28-32.

52. Ochiai N, Nishizuka M, Osada S, Imagawa M (2016) Fad24, a Positive Regulator of Adipogenesis, Is Required for S Phase Re-entry of C2C12 Myoblasts Arrested in G0 Phase and Involved in p27(Kip1) Expression at the Protein Level. Biol Pharm Bull 39(5): 807-814.

53. Honey S, Futcher B (2007) Roles of the CDK phosphorylation sites of yeast Cdc6 in chromatin binding and rereplication. Mol Biol Cell 18(4): 1324-1336.

54. Zhai Y, Yung PYK, Liang C (2011) Cell cycle control of DNA replication by phosphorylation and dephosphorylation of replication-initiation proteins in budding yeast. In: J Kušić-Tišma (Ed.), Fundamental Aspects of DNA Replication. Chapter 6, Tech Publisher, pp. 87-106.

55. Du YC, Stillman B (2002) Yph1p, an ORC-interacting protein: potentia links between cell proliferation control, DNA replication, and ribosome biogenesis. Cell 109(7): 835-48.

56. Galani K, Nissan TA, Petfalski E, Tollervey D, Hurt E, (2004) Rea1, a dynein-related nuclear AAA-ATPase, is involved in late rRNA processing and nuclear export of 60 S subunits. J Biol Chem 279(53): 55411-55418.
57. Krogan NJ, Peng WT, Cagney G, Robinson MD, Haw R, et al. (2004) High-definition macromolecular composition of yeast RNA-processing complexes. Mol Cell 13(2): 225-239.

58. Nissan TA, Galani K, Maco B, Tollervey D, Aebi U, et al. (2004) A preribosome with a tadpole-like structure functions in ATP-dependent maturation of 60S subunits. Mol Cell 15(2): 295-301.

59. Saka Y, Esashi F, Matsusaka T, Mochida S, Yanagida M (1997) Damage and replication checkpoint control in fission yeast is ensured by interactions of Crb2, a protein with BRCT motif, with Cut5 and Chk1. Genes Dev 11(24): 3387-400.

60. Zegerman P, Diffley JFX (2007) Phosphorylation of Sld2 and Sld3 by cyclin-dependent kinases promotes DNA replication in budding yeast. Nature 445(7125): 281-285.

61. Tanaka S, Umemori T, Hirai K, Muramatsu S, Kamimura Y, et al. (2007) CDK-dependent phosphorylation of Sld2 and Sld3 initiates DNA replication in budding yeast. Nature 445(7125): 328-332.

62. Liu J, Smith CL, DeRyckere D, DeAngelis K, Martin GS, et al. (2000) Structure and function of Cdc6/Cdc18: implications for origin recognition and checkpoint control. Mol Cell 6(3): 637-648.

63. Ohta S, Koide M, Tokuyama T, Yokota N, Nishizawa S, et al. (2001) Cdc6 expression as a marker of proliferative activity in brain tumors. Oncol Rep 8(5): 1063-1066.

64. Robles LD, Frost AR, Davila M, Hutson AD, Grizzle WE, et al. (2002) Down-regulation of $\mathrm{Cdc} 6$, a cell cycle regulatory gene, in prostate cancer. J Biol Chem 277(28): 25431-25438.

65. Randell JC, Bowers JL, Rodriguez HK, Bell SP (2006) Sequential ATP hydrolysis by Cdc6 and ORC directs loading of the Mcm2-7 helicase. Mol Cell 21(1): 29-39.

66. Yoshida K, Sugimoto N, Iwahori S, Yugawa T, Narisawa-Saito M, et al. (2010) CDC6 interaction with ATR regulates activation of a replication checkpoint in higher eukaryotic cells. Journal of Cell Science 123(Pt 2): 225-235.

67. Nishitani H, Nurse P (1995) p65cdc18 plays a major role controlling the initiation of DNA replication in fission yeast. Cell 83(3): 397-405.

68. Piatti S, Lengauer C, Nasmyth K (1995) Cdc6 is an unstable protein whose de novo synthesis in G1 is important for the onset of $S$ phase and for preventing a 'reductional' anaphase in the budding yeast Saccharomyces cerevisiae. ЕМBO J 14(15): 3788-3799.

69. Elsasser S, Chi Y, Yang P, Campbell JL (1999) Phosphorylation controls timing of Cdc6p destruction: a biochemical analysis. Mol Biol Cell 10(10): 3263-3277.

70. Sanchez M, Calzada A, Bueno A (1999) The Cdc6 protein is ubiquitinated in vivo for proteolysis in Saccharomyces cerevisiae. J Biol Chem 274(13): 9092-9097.

71. Speck C, Chen Z, Li H, Stillman B (2005) ATPase-dependent cooperative binding of ORC and Cdc6 to origin DNA. Nat Struct Mol Biol 12(11): 965971.

72. Speck C, Stillman B (2007) Cdc6 ATPase Activity Regulates ORC-CDC6 Stability and the Selection of Specific DNA Sequences as Origins of DNA Replication. J Biol Chem 282(16): 11705-11714.

73. Hofmann JF, Beach D (1994) cdt1 is an essential target of the Cdc10/Sct1 transcription factor: requirement for DNA replication and inhibition of mitosis. EMBO J 13(2): 425-434.

74. Nishitani H, Lygerou Z, Nishimoto T, Nurse P (2000) The Cdt1 protein is required to license DNA for replication in fission yeast. Nature 404(6778): 625-628.

75. Tanaka S, Diffley JF (2002) Interdependent nuclear accumulation of budding yeast Cdt1 and Mcm2-7 during G1 phase. Nat Cell Biol 4(3): 198-207.

76. Devault A, Vallen EA, Yuan T, Green S, Bensimon A, et al. (2002) Identification of Tah11/Sid2 as the ortholog of the replication licensing factor Cdt1 in Saccharomyces cerevisiae. Curr Biol 12(8): 689-694. 
77. Wohlschlegel JA, Dwyer BT, Dhar SK, Cvetic C, Walter JC, Dutta A, et al. (2000) Inhibition of eukaryotic DNA replication by geminin binding to Cdt1. Science 290(5500): 2309-2312.

78. Evrin C, Clarke P, Zech J, Lurz R, Sun J, et al. (2009) A double-hexameric MCM2-7 complex is loaded onto origin DNA during licensing of eukaryotic DNA replication. Proc Natl Acad Sci USA 106(48): 2024020245.

79. Remus D, Beuron F, Tolun G, Griffith JD, Morris EP, et al. (2009) Concerted loading of Mcm2-7 double hexamers around DNA during DNA replication origin licensing. Cell 139(4): 719-730.

80. Maine GT, Sinha P, Tye BK (1984) Mutants of $S$. cerevisiae defective in the maintenance of minichromosomes. Genetics 106(3): 365-385.

81. Tye BK (1999) MCM proteins in DNA replication. Annu Rev Biochem 68: 649-686.

82. Yu Z, Feng D, Liang C (2004) Pairwise interactions of the six human MCM protein subunits. J Mol Biol 340(5): 1197-206.

83. Labib K, Tercero J, eacute A, Diffley JF, nbsp X (2000) Uninterrupted MCM2-7 Function Required for DNA Replication Fork Progression. Science 288(5471): 1643-1647.

84. Aparicio OM, Weinstein DM, Bell SP (1997) Components and dynamics of DNA replication complexes in $S$. cerevisiae: Redistribution of MCM proteins and Cdc45p during S phase. Cell 91(1): 59-69.

85. Moyer SE, Lewis PW, Botchan MR (2006) Isolation of the Cdc45/Mcm2$7 /$ GINS (CMG) complex, a candidate for the eukaryotic DNA replication fork helicase. Proc Natl Acad Sci U S A 103(27): 10236-10241.

86. Ilves I, Petojevic T, Pesavento JJ, Botchan MR (2010) Activation of the MCM2-7 helicase by association with Cdc45 and GINS proteins. Mol Cell 37(2): 247-258.

87. Liku ME, Nguyen VQ, Rosales AW, Irie K, Li JJ (2005) CDK phosphorylation of a novel NLS-NES module distributed between two subunits of the Mcm2-7 complex prevents chromosomal rereplication. Mol Biol Cell 16(10): 5026-5039.

88. Nguyen VQ, Co C, Irie K, Li JJ (2000) Clb/Cdc28 kinases promote nuclear export of the replication initiator proteins Mcm2-7. Curr Biol 10(4): 195-205.

89. Davey M J, Indiani C, O’Donnell M (2003) Reconstitution of the Mcm2-7p heterohexamer, subunit arrangement, and ATP site architecture. J Biol Chem 278(7): 4491-4499.

90. Aladjem M (2007) Replication in context: dynamic regulation of DNA replication patterns in metazoans. Nature Reviews Genetics 8(8): 588600.

91. Tanaka S, Araki H (2013) Helicase Activation and Establishment of Replication Forks at Chromosomal Origins of Replication. Cold Spring Harb Perspect Biol 5(12): a010371.

92. Douglas ME, Ali FA, Costa A, Diffley JFX (2018) The mechanisms of eukaryotic CMG helicase activation. Nature 555: 265-268.

93. Homesley L, Lei M, Kawasaki Y, Sawyer S, Christensen T, et al. (2000) Mcm10 and the MCM2-7 complex interact to initiate DNA synthesis and to release replication factors from origins. Genes Dev 14(8): 913-926.

94. Baxley RM, Bielinsky AJ (2017) Mcm10: A Dynamic Scaffold at Eukaryotic replication Forks. Genes 8(2): 73.

95. Ricke RM, Bielinsky AK (2004) Mcm10 regulates the stability and chromatin association of DNA polymerase- $\alpha$. Mol Cell 16(2): 173-185.

96. Labib K, Kearsey SE, Diffley JFX (2001) MCM2-7 Proteins Are Essential Components of Prereplicative Complexes that Accumulate Cooperatively in the Nucleus during G1-phase and Are Required to Establish, But Not Maintain, the Sphase Checkpoint. Mol Biol Cell 12(11): 3658-3667.

97. Sawyer SL, Cheng IH, Chai W, Tye BK (2004) Mcm10 and Cdc45 cooperate in origin activation in Saccharomyces cerevisiae. J Mol Biol 340(2): 195202 .

98. Gambus A, Jones RC, Sanchez-Diaz A, Kanemaki M, van Deursen F Edmondson RD, Labib K, et al. (2006) GINS maintains association of Cdc45 with MCM in replisome progression complexes at eukaryotic DNA replication forks. Nat Cell Biol 8(4): 358-366.

99. Bruck I, Kaplan DL (2011) Origin single-stranded DNA releases Sld3 from Mcm2-7, allowing GINS to bind Mcm2-7. J Biol Chem 286(21): 18602-18613.

100. Petropoulou C, Kotantaki P, Karamitros D, Taraviras S (2008) Cdt1 and Gemini in cancer markers or triggers of malignant transformation? Front Biosci 13: 4485-4494.

101. Liontos M, Koutsami M, Sideridou M, Evangelou K, Kletsas D, et al. (2007) Deregulated overexpression of hCdt1 and hCdc6 promotes malignant behavior. Cancer Res 67(22): 10899-10909.

102. Tsaniras SC, Kanellakis N, Symeonidou IE, Nikolopoulou P, Lygerou $\mathrm{Z}$, et al. (2014) Licensing of DNA replication, cancer, pluripotency and differentiation: An interlinked world? MSeminars in Cell \& Devlopmental Biology 30: 174-180.

103. Feng D, Tu Z, Wu W, Liang C (2003) Inhibiting the expression of DNA replication initiation proteins induces apoptosis in human cancer cells. Cancer Res 63(21): 7356-7364.

104. Chen Z, Speck C, Wendel P, Tang C, Stillman B, et al. (2008) The architecture of the DNA replication origin recognition complex in Saccharomyces cerevisiae. Proc Natl Acad Sci USA 105(30): 1032610331.

105. Huang Y, Amin A, Qin Y, Wang Z, Jiang H, et al. (2016) A Role of hIPI3 in DNA Replication Licensing in Human Cells. PLoS ONE 11(4): e0151803.

106. Lee DG, Bell SP (1997) Architecture of the yeast origin recognition complex bound to origins of DNA replication. Mol Cell Biol 17(12): 7159-7168.

107. Liang C, Weinreich M, Stillman B (1995) ORC and Cdc6p interact and determine the frequency of initiation of DNA replication in the genome. Cell 81(5): 667-676.

108. Lõoke M, Reimand J, Sedman T, Sedman J, Järvinen L, et al. (2010) Relicensing of transcriptionally inactivated replication origins in budding yeast. J Biol Chem 285(51): 40004-40011.

109. Santocanale C, Diffley JF (1996) ORC- and Cdc6-dependent complexes at active and inactive chromosomal replication origins in Saccharomyces cerevisiae. EMBO J 15(23): 6671-6679.

110. Shen Z, Sathyan KM, Geng Y, Zheng R, Chakraborty A, et al. (2010) A WD-repeat protein stabilizes ORC binding to chromatin. Mol Cell 40(1): 99-111.

111. Weinreich M, Liang C, Chen HH, Stillman B (2001) Binding of cyclindependent kinases to ORC and Cdc6p regulates the chromosomes replication cycle. Proc Natl Acad Sce USA 98(20): 11211-11217.

112. Wilmes GM, Bell SP (2002) The B2 element of the Saccharomyces cerevisiae ARS1 origin of replication requires specific sequences to facilitate pre-RC formation. Proc Natl Acad Sci USA 99(1): 101-106.

113. Yan S, Willis J (2013) WD40-repeast protein WDR18 collaborates with TopBp1 to facilitate DNA damage checkpoint signaling. Biochemical and biophysical research communications 431(3): 466-471. 


\section{ISSN: 2574-1241}

DOI: 10.26717/BJSTR.2019.23.003830

Chun Liang. Biomed J Sci \& Tech Res

\section{(c) (P) This work is licensed under Creative}

Submission Link: https://biomedres.us/submit-manuscript.php

\begin{tabular}{ll} 
BIOMEDICAL & Assets of Publishing with us \\
RESEARCHES & - Global archiving of articles \\
& - Immediate, unrestricted online access \\
\hline
\end{tabular}

